\title{
Dance and Science: an analysis of the creative process behind "Transitions", a show by Contemporary Dance Company of UFRJ
}

\author{
André Meyer Alves de Lima, Danielle Vianna Menezes Pinto Almeida, \\ Adalberto Ramon Vieyra
}

Federal University of Rio de Janeiro, Brazil

\begin{abstract}
This study proposes an esthetic reflection on the influence of the biological sciences on dance as a variety of contemporary art. The relationship between science and art in this study is grounded on François Dagognet's hyperphenomenology, particularly his representation of the major role played by the image-symbol as essential base of present-day scientific activity [1]. Contemporary dance creators and companies such as Wayne Mac Gregor, Phylobolus Dance Company and Liz Lerman Dance Exchange are analyzed, in order to highlight how some biosciences themes are explored as creative potentials in their dance performances [2,3,4]. We aim at relating biological shapes and patterns existing in the cellular and molecular universe with the fundamentals of dance described by Helenita Sá Earp. Earp's conception posits dance as wide-scoped knowledge, including a "science of dance" where intuition works as basic foundation and its epistemology is made of an open type of knowledge named "dance parameters", namely motion, space, shape, dynamics and time. These parameters allow establishing access points for multiple connections within the realm of dance. This approach tends to generate a kind of access to body actions stimulating interdisciplinarity between several areas of knowledge [4]. As study-case, we focus methodologically on the processes of esthetic creation involved in the production of a multimedia show entitled "Transitions" by appealing to biochemical notions, especially some features of cytology and cellular morphology, cell proliferation and processes of cellular transportation [5].
\end{abstract}

Keywords: Arts; Dance; Science; Biochemistry; Helenita Sá Earp.

References:

[1] Bulcão M. The Enjoyment of Knowledge and Imagination: François Dagonet on Science and Contemporary Art. Rio de Janeiro: Mauad; 2010.

[2] Lerman ZM. Chemistry: An Inspiration for Theatre and Dance. Chemical Education International [online]. 2005 Sep 26 [cited 2011 Mar 08];6(1):[about 5 p.]. Available from:

http://old.iupac.org/publications/cei/vol6/11_Lerman.pdf

[3] Burg J, Lüttringhaus K. Entertaining with Science, Educating with Dance. Computers in Entertainment (CIE) [serial online]. 2006 Apr-Jun [cited 2011 Mar 10];4(2):[about 14 p.]. Available from:

http://dblp.uni-trier.de/db/journals/cie/cie4.html 
[4] Lima AMA. Biological Sciences in contemporary choreographic scene: an analysis from the epistemology of François Dagognet. In: VI Congresso de pesquisa e pós-graduação em Artes Cênicas; 2010 Nov 9-12; São Paulo. Porto Alegre (Brazil): Memória ABRACE Digital; c2010. 7 p. Available from: http://portalabrace.org/memoria1/?p=1224

[5]__. Reflections on the creation and use of dance performance with interactive electronic media for diffusion of Biochemistry. In: V Congresso de pesquisa e pós-graduação em Artes Cênicas; 2008 Out 28-31; Belo Horizonte. Porto Alegre (Brazil): Memória ABRACE Digital; c2008. Available from: http://www.portalabrace.org/vcongresso/resumospesquisadanca.html

\section{Dança e ciência: uma análise dos processos de criação do espetáculo "Transições" da Companhia de Dança Contemporânea da UFRJ}

\section{RESUMO}

A proposta desta pesquisa é de refletir estéticamente sobre a influência das ciências biológicas na dança como forma de arte contemporânea. Relacionamos ciência e arte a partir da hiperfenomenologia de François Dagognet nos sentido de mostrar o papel primordial que faz da imagem-símbolo o fundamento primordial da atividade científica da atualidade[1]. Fazemos um análise de criadores e companhias de dança contemporânea, como por exemplo: Wayne Mac Gregor, a Phylobolus Dance Company e a Liz Lerman Dance Exchange no sentido de evidenciar de que forma são explorados temas presentes nas biociências como potencial criador em seus espetáculos de dança[2,3,4]. Neste contexto, buscamos relacionar formas e padrões biológicos presentes no universo celular e molecular com os Fundamentos da Dança propostos por Helenita Sá Earp. A concepção de Earp propõe a dança como conhecimento amplo, isto é, que envolva uma "ciência da dança" cuja intuição seja o fundamento básico e que sua epistemologia seja feita por conhecimentos abertos denominados Parâmetros da Dança: Movimento, Espaço, Forma, Dinâmica e Tempo. Parâmetros que permitem estabelecer pontos de entrada para o estabelecimento de coneções múltiplas na dança. Esta compreessão tende a gerar uma espécie de acesso as ações corporais que estimula a interdisciplinaridade entre diferentes áreas do conhecimento[4]. Como estudo de caso, enfocamos metodologicamente processos de criação estética na montagem de um espetáculo multimídia de dança intitulado "Transições" a partir de noções advindas da bioquímica, notadamente, em aspectos da citologia e morfologia celular, proliferação celular e processos celulares de transporte[5].

Palavras-Chave: Arte; Dança; Ciências; Bioquímica; Helenita Sá Earp.

(c)) BY-NC-ND Licensed to GIRI

Support: authors declare that this study received funding

Conflict of interest: authors declare there is no conflict of interest

Correspondence author: André Meyer Alves de Lima, amlima@bioqmed.ufrj.br

How to cite this article: de Lima AMA, Almeida DVMP, Vieyra AR. Dance and Science: an analysis of the creative process behind "Transitions", a show by Contemporary Dance Company of UFRJ. Int J High Dilution Res [online]. 2011 [cited YYYY Month dd]; 10(36): 292-293. Proceedings of the XXV GIRI Symposium and VIII CBFH; 2011 Sep 04-07; Foz do Iguaçu (Brazil). GIRI and ABFH; 2011; Available from: http://www.feg.unesp.br/ ojs/index.php/ijhdr/article/view/516/539 